\title{
Fault Diagnosis of Wind Turbine Blades Using Histogram Features through Nested Dichotomy Classifiers
}

\author{
A. Joshuva, S. Sivakumar, R. Sathishkumar, \\ G. Deenadayalan, R. Vishnuvardhan
}

\begin{abstract}
This study makes an attempt of classifying different fault conditions which occurs on wind turbine blade due to environmental stress and high wind speed. Here three bladed horizontal axis variable wind turbine was used for experimental study and different faults like blade crack, hub-blade loose connection, erosion, pitch angle twist and blade bend was considered. This study had been carried out in three phases namely feature extraction, feature selection and feature classification. Initially vibration signals are noted for different blade conditions and required features are obtained using histogram features. Secondly, from the extracted feature, most dominating feature need to be chosen using $\mathrm{J48}$ decision tree classifier. Later, the selected feature is fed into the classifiers like Nested Dichotomy (ND), Class-Balanced Nested Dichotomies (CBND) and Data near Balanced Nested Dichotomy (DNBND) for classification of the faults. These classifiers are compared with respect to their accuracy to suggest a better model for fault diagnosis on blade. The suggested model can be incorporated in real-time system to monitor the condition of wind turbine blade.

Keywords--- Fault Diagnosis, Wind Turbine Blade, Condition Monitoring, Histogram Features, Vibration Signals, Nested Dichotomy Classifiers.
\end{abstract}

\section{INTRODUCTION}

Renewable energy sources have drawn in much consideration because of their positive influence on society and environment. "Wind energy is a rapid growing clean renewable energy source [1].

Wind turbines are actuality produced to upgrade operational implementation and yield through expanding blade size and enhancing physical framework. It has been confirmed that blade damage may bring about lack in productivity, and its repair is expensive and requires considerable repair time.

To avoid extreme blade damage and minor failures, it is important to identify the early failures by routine monitoring, and proceeding with evaluation of the structural health of wind turbine blades [2]. The objective of this study is to build up a model for the condition monitoring of wind turbine blade using machine learning approach."

Manuscript received September 16, 2019.

A. Joshuva*, Centre for Automation and Robotics (ANRO), Department of Mechanical Engineering, Hindustan Institute of Technology and Science, Old Mahabalipuram Road, Padur, Kelambakam, Chennai, Tamil Nadu. (e-mail: joshuva1991@gmail.com)

S. Sivakumar, Department of Mechanical Engineering, Hindustan Institute of Technology and Science, Old Mahabalipuram Road, Padur, Kelambakam, Chennai, Tamil Nadu.

R. Sathishkumar, Department of Automobile Engineering, Hindustan Institute of Technology and Science, Old Mahabalipuram Road, Padur, Kelambakam, Chennai, Tamil Nadu.

G. Deenadayalan, Centre for Automation and Robotics (ANRO), Department of Mechanical Engineering, Hindustan Institute of Technology and Science, Old Mahabalipuram Road, Padur, Kelambakam, Chennai, Tamil Nadu.

R. Vishnuvardhan, Department of Mechatronics Engineering, Sri Krishna College of Engineering and Technology, Coimbatore, Tamil Nadu.
There are two types of approaches which are carried out on condition monitoring assessment they are traditional approach and machine learning approach. "The traditional approach is mainly used for the stationary signals where frequency of the signals component does not change with respect to time but rotating machines produce non-stationary signals [3].

Since the frequency components change due to wear and tear, fault discrimination is very difficult in traditional approach. Hence, not preferred. In machine learning approach, algorithms have capability to learn continuously and adapt themselves to the varying situations. Researches often resort to machine learning approach for fault diagnosis of mechanical systems [4]."

Many studies were carried out using machine learning studies and simulation studies to name a few, Andrew Kusiak and Anoop Verma [5] built a data-driven model for monitoring blade pitch faults in wind turbines. "They considered two blade pitch faults namely, blade angle asymmetry and blade angle implausibility and determine the associations between them.

They conducted the study using bagging (72.5\%), artificial neural network (76.2\%), pruning rule-based classification tree $(75.5 \%)$, K-nearest neighbour $(73.5 \%)$ and genetic programming $(\mathbf{7 4 . 7 \% )}$ algorithms. They considered only pitch fault and other faults were not considered in their study."

Abouhnik and Albarbar [6] simulated crack in wind turbine blades and carried out crack location prediction study using vibration measurements and the level of an empirically decomposed feature intensity level (EDFIL). "The main drawback in this study is they do not consider other fault parameters in their study expect crack. A classification and detection of wind turbine pitch faults were done by Godwin and Matthews [7]. They used RIPPER algorithm in their study. They diagnosed the electrical control system faults, in particular, wind turbine pitch faults which yielded them $87.05 \%$ classification accuracy. They considered pitch faults alone in this study.

A study on integrating structural health management with contingency control for wind turbines using nonlinear high fidelity simulation was carried out by Frost et al., [8]. They studied about the structural health of the blade, the speed of the turbine and decision making using prognostic information and achieved $90 \%$ accuracy in their work. They have done the study for blade bend fault; and not considered other faults.

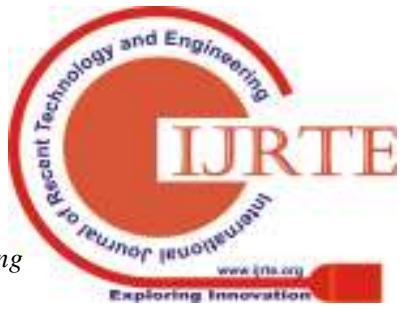




\section{FAULT DIAGNOSIS OF WIND TURBINE BLADES USING HISTOGRAM FEATURES THROUGH NESTED DICHOTOMY CLASSIFIERS}

Chen et al., [9] conducted an experiment on wind turbine pitch faults prognosis using apriori knowledge based adaptive neuro-fuzzy inference system (ANFIS) and SCADA data. They obtained $88.30 \%$ classification accuracy for the blade pitch fault. In this study also they considered pitch fault alone."

A work on structural health monitoring of wind turbine blades with acoustic source localization using wireless sensor networks was done by Bouzid et al., [10]. "They done the online monitoring of the blade for the fault identification of erosion and crack which affects the blade using acoustic emission NDT. They predicted the error rate of $7.98 \%$. Lee et al., [11] done a work on wind turbine blade moment signals to blade condition monitoring using a transformation algorithm. They presented a novel method of transforming blade moment signals on a horizontal axis 3blade wind turbine.

Herath et al., [12] conducted a study on the design of shape adaptive wind turbine blades using differential stiffness bend twist (DSBT) coupling using FEA analysis. They proposed this concept to control the deformation behaviour in the blades. Liu et al., [13] carried out a study on the influence of alternating loads on nonlinear vibration characteristics of cracked blade in a rotor system using FEM analysis. They did the experiment for different alternating loads for the identification of the crack fault and other faults not taken into consideration."

Numerous works were carried out using simulation studies however, only a very few of the experimental studies for condition monitoring on wind turbine blade was carried out. Some researchers carried out fault diagnosis system using machine learning approach and they considered very few faults in their study. "Only selected algorithms were used to perform the fault prediction on wind turbine blade and many algorithms are yet to be studied [14]. Figure 1 shows the methodology of the work done. The contribution of the present study.

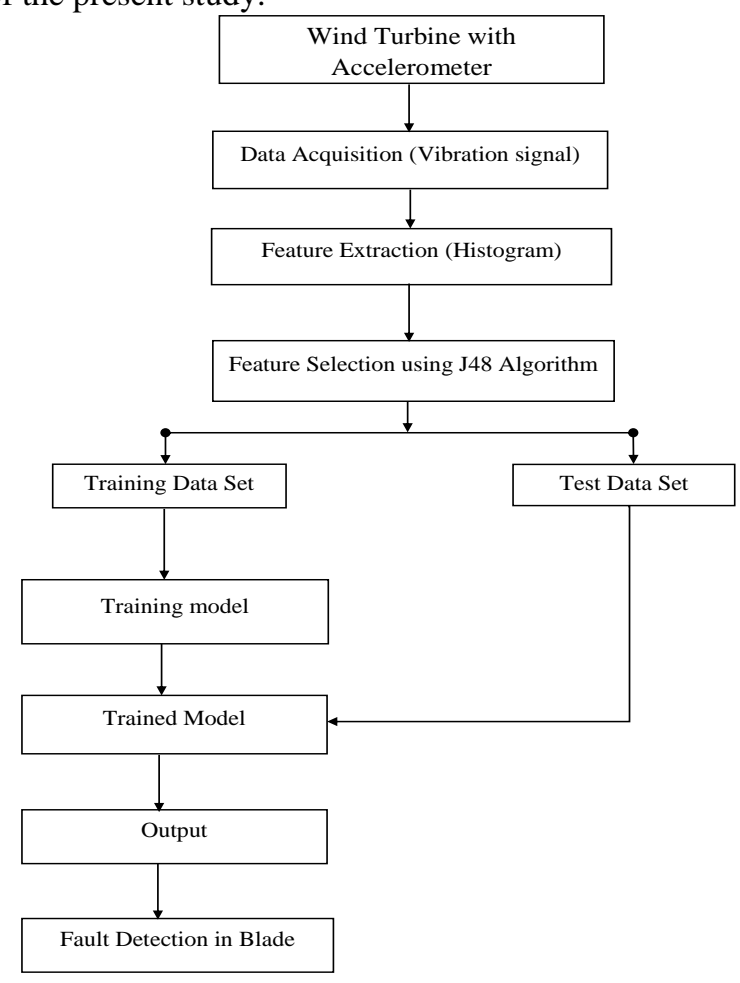

Figure 1: Methodology
1. This study considers more than two faults for fault diagnosis of wind turbine (blade crack, erosion, hub-blade loose connection, pitch angle twist and blade bend).

2. This problem is modelled as a multiclass classification problem and attempts to classify using classifiers such as Nested Dichotomy (ND), Class-Balanced Nested Dichotomies (CBND) and Data near Balanced Nested Dichotomy (DNBND).

\section{EXPERIMENTAL STUDIES}

The main aim of this study is to classify whether the blades are in good condition or in a defective state. "If it is defective, then the objective is to identify the type of fault. The experimental setup and experimental procedure are described in the following subsections.

\section{Experimental Setup}

The experiment was carried out on a $50 \mathrm{~W}, 12 \mathrm{~V}$ variable speed wind turbine (MX-POWER, model: FP-50W-12V). The technical parameters of a wind turbine are given in Table 1. The wind turbine was mounted on a fixed steel stand in front of the open circuit wind tunnel outlet.

The wind tunnel speed ranges from $5 \mathrm{~m} / \mathrm{s}$ to $15 \mathrm{~m} / \mathrm{s}$ and acts as a wind source to start the wind turbine. The wind speed was varied continuously in order to simulate the environmental wind condition. The experimental setup is shown in Figure 2.

Piezoelectric type accelerometer was used as a transducer for acquiring vibration signals. It has high sensitivity for detecting faults.

Hence, accelerometers are widely used in condition monitoring. In this case, an uniaxial accelerometer of $500 \mathrm{~g}$ range, $100 \mathrm{mV} / \mathrm{g}$ sensitivity, and resonant frequency around $40 \mathrm{~Hz}$ was used.

Table 1: Technical Parameters of Wind Turbine

\begin{tabular}{|l|l|}
\hline Model & FP-50W-12V \\
\hline Rated Power & $50 \mathrm{~W}$ \\
\hline Rated Voltage & $12 \mathrm{~V}$ \\
\hline Maximum Current & $4 \mathrm{~A}$ \\
\hline Rated Rotating Rate & $850 \mathrm{rpm}$ \\
\hline Start-up Wind Speed & $2.5 \mathrm{~m} / \mathrm{s}$ \\
\hline Cut-in Wind Speed & $3.5 \mathrm{~m} / \mathrm{s}$ \\
\hline Cut-out Wind Speed & $15 \mathrm{~m} / \mathrm{s}$ \\
\hline Security Wind Speed & $40 \mathrm{~m} / \mathrm{s}$ \\
\hline Rated Wind Speed & $12.5 \mathrm{~m} / \mathrm{s}$ \\
\hline Engine & $\begin{array}{l}\text { Three- } \\
\text { phase permanent magnet generator }\end{array}$ \\
\hline Rotor Diameter & $1050 \mathrm{~mm}$ \\
\hline Blade Material & Carbon fiber reinforced plastics \\
\hline
\end{tabular}

The piezoelectric accelerometer (DYTRAN 3055B1) was mounted on the nacelle near to the wind turbine hub to record the vibration signals using an adhesive mounting technique.

It was connected to the DAQ system through a cable. The data acquisition system (DAQ) used was NI USB 4432 model. The DAQ card has five analogue input channels with 
a sampling rate of 102.4-kilo samples per second with 24bit resolution. The accelerometer is coupled to a signal conditioning unit which consists of an inbuilt charge amplifier and an analogue-to-digital converter (ADC).

From the ADC, the vibration signal was taken. These vibration signals were used to extract features through feature extraction technique.

One end of the cable is plugged to the accelerometer and the other end to the AIO port of DAQ system. NI LabVIEW was used to interface the transducer signal and the system (PC).

\section{Experimental Procedure}

In the present study, three-blade variable horizontal axis wind turbine (HAWT) was used.

Initially, the wind turbine was considered to be in good condition (free from defects, new setup) and the signals were recorded using an accelerometer.

These signals were recorded with the following specifications.

1. Sample length: The sample length was chosen long enough to ensure data consistency; and also the following points were considered. ARMA measures are more meaningful when the number of samples is sufficiently large. On the other hand, as the number of samples increases the computation time increases. To strike a balance, a sample length of 10000 was chosen.

2. Sampling Frequency: The sampling frequency should be at least twice the highest frequency contained in the signal as per Nyquist sampling theorem. By using this theorem sampling frequency was calculated as $12 \mathrm{kHz}(12000 \mathrm{~Hz})$.

3. Number of samples: Minimum of 100 (hundred) samples were taken for each condition of the wind turbine blade and the vibration signals were stored in data files.

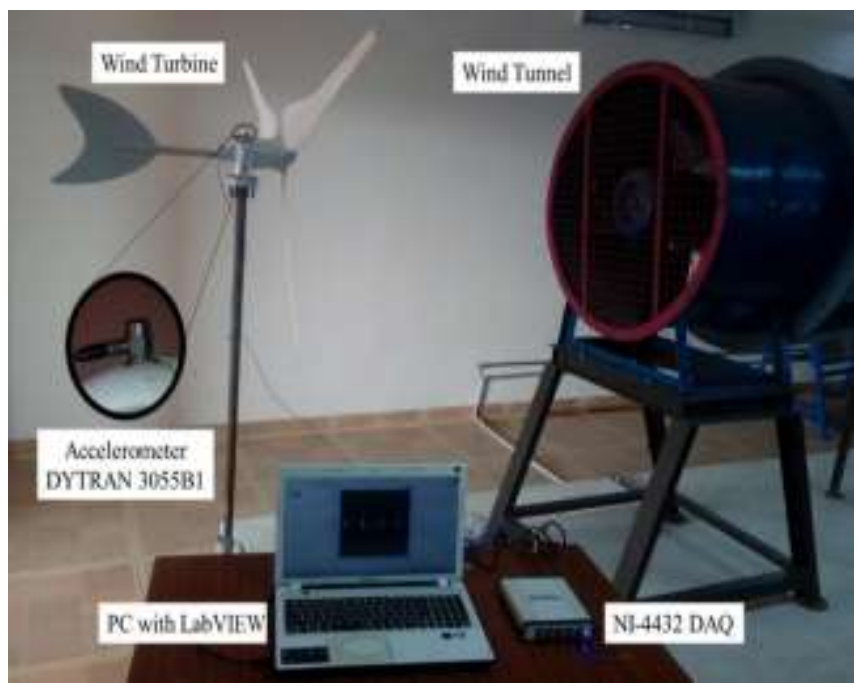

Figure 2: Wind Turbine Setup

The following faults were simulated one at a time while all other components remain in good condition and the corresponding vibration signals were acquired. Figure 3 shows the different blade fault conditions which are simulated on the blade.

a) Blade bend (BB): This fault occurs due to the high-speed wind and complex forces caused by the wind. The blade was made to flap wise bend with $10^{\circ}$ angle.

b) Blade crack (BC-2): This occurs due to foreign object damage on the blade while it is in operating condition. On the blade, $15 \mathrm{~mm}$ crack was made.

c) Blade erosion (BE): This fault is due to the erosion of the top layer of the blade by the highspeed wind. The smooth surface of the blade was eroded using emery sheet $(320 \mathrm{Cw})$ to provide an erosion effect on the blade.

d) Hub-blade loose contact: This fault generally occurs on a wind turbine blade due to an excessive runtime or usage time. The bolt connecting the hub and blade was made loose to obtain this fault.

e) Blade pitch angle twist (PAT): This fault occurs due to the stress on the blade caused by high-speed wind. This makes the pitch get twisted, creating a heavy vibration to the framework. To attain this fault, blade pitch was twisted about $12^{\circ}$ angle with respect to the normal blade condition.

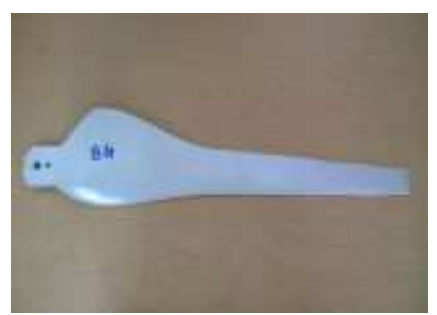

Good condition blade

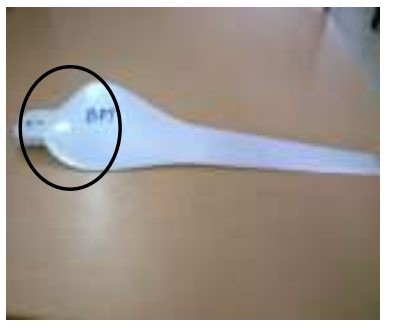

Blade with pitch angle twist

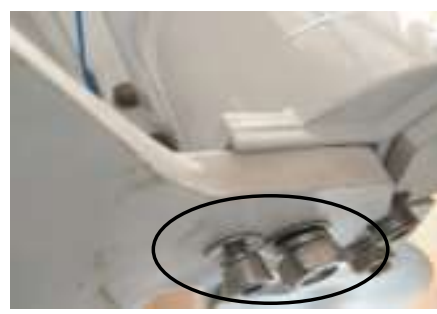

Hub-blade loose connection

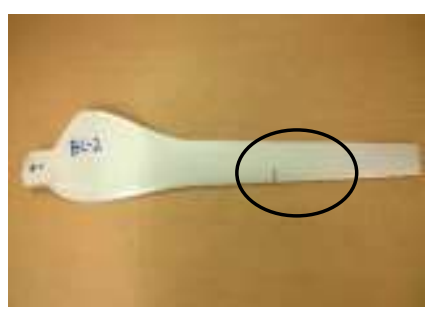

Blade with crack

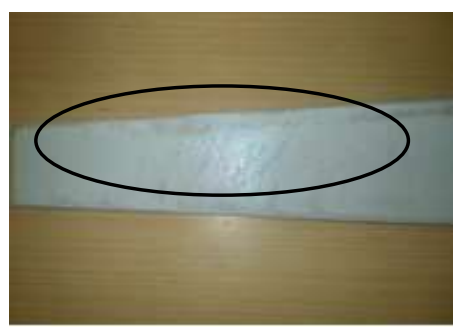

Blade with erosion

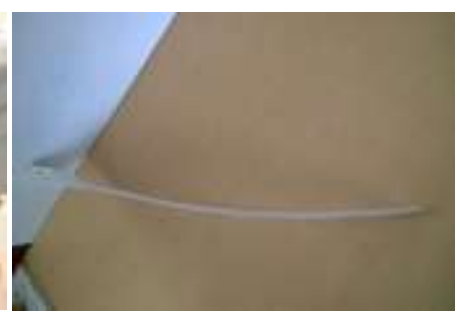

Blade with bend (Top View)
Figure 3: Various Blade Fault Conditions 

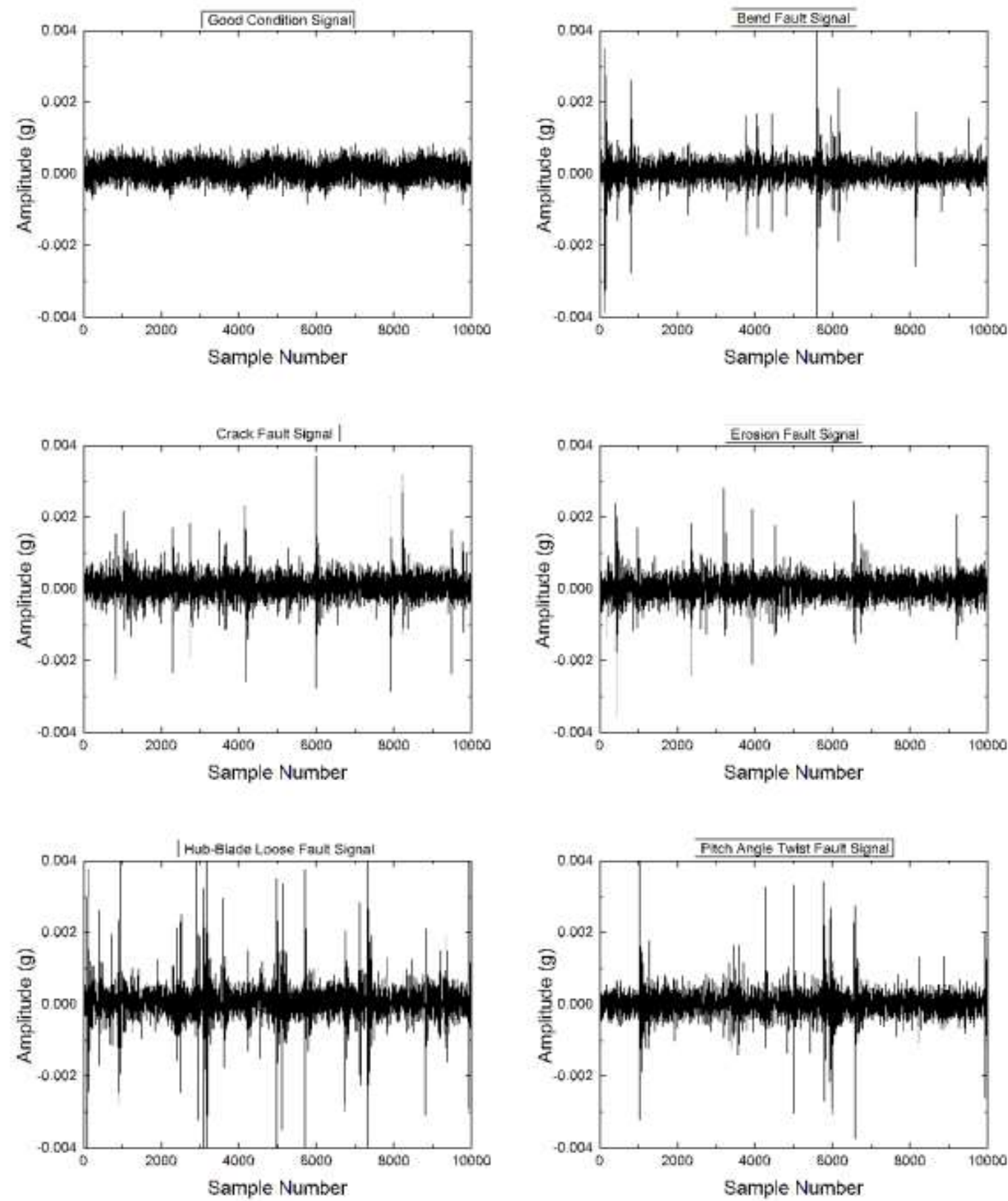

Figure 4: Vibration Signal Plot

In Figure 4, the vibration signals (sample number vs. amplitude) are shown which were taken for different conditions of the wind turbine blade (good condition blade, blade bend, blade erosion, hub-blade loose connection, blade crack and pitch angle twist).

\section{FEATURE EXTRACTION}

The vibration signals were obtained for good and other faulty conditions of the blades. If the time domain sampled signals are given directly as inputs to a classifier, then the number of samples should be constant. The number of samples obtained are the function of rotation of the blade speed. "Hence, it cannot be used directly as the input to the classifier. However, a few features must be extracted before the classification process. The histogram was used as a feature extracting tool in this study. From the noted vibration signals, the needed feature is taken and that features are denoted as histogram features. There are two main factors to be considered in selection of bins they are, bin range and bin width [15].

The bin range must be from lowest of minimum amplitude $(-0.017988)$ to extreme of maximum amplitude $(0.024833)$ of all the six classes (good, bend, crack, erosion, loose and PAT). The number of bins for the fault diagnosis of wind turbine blade has been attained by carrying out a sequence of trials using $\mathrm{J} 48$ algorithm with different number of bins. Initially, the range of bin is separated into two equivalent portions. That is to say, the number of bins utilized is two. The two histogram features, to be specific,

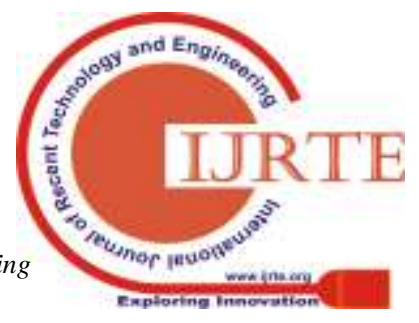


$\mathrm{X} 1$ and $\mathrm{X} 2$ are extracted and the relating classification accuracy is additionally acquired by using J48 algorithm.

The approach and methodology of performing the same using J48 algorithm are clarified in Section 4. A set of related trails is done with various numbers of bins from 2, 3, $4,5, \ldots, 100$ and the corresponding results are shown in figure 5 .

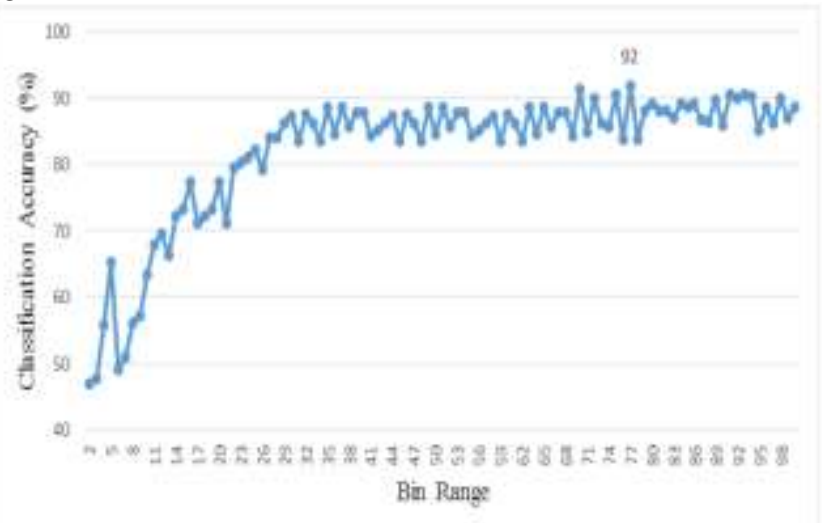

Figure 5: Bin Range vs. Classification Accuracy

From figure 5, bin size 77 has been chosen since the classification accuracy of bin 77 was found to be $92 \%$. A set of 77 starting from X1, X2 .. X77 were extracted from the vibration signals and these are denoted as histogram features.

The amplitude ranges from -0.017988 to 0.024833 . For further study, rather than utilizing vibration signals directly, the histogram features extracted from vibration signals are utilized.

The procedure of calculating applicable parameters of the signals that represent the data contained in the signal is called feature extraction. Histogram analysis of vibration signals yields distinctive parameters."

All the extracted histogram features, X1 to X77 extracted from the vibration signals may not contain the needed information for classification. The applicable ones are selected using J48 algorithm.

\section{FEATURE SELECTION}

Once the bin size 77 is chosen, the applicable features are to be selected from the bin since some features will be less predicting the faults. "For feature extraction, J48 algorithm is used where they predict the classes in a tree structure [16].

A typical tree comprises of a number of branches, one root, a number of nodes and a number of leaves. One branch is a chain of nodes from the root to a leaf; and each node involves one feature. The occurrence of a feature in a tree delivers the data about the significance of the accompanying feature.

J48 algorithm provides the information of most contributing features in the bin via top-down tree structure. Features that have less discriminating capability can be deliberately rejected by fitting the threshold.

This conception makes use of in choosing good features by the algorithm by inbuilt nature. Features which have good discriminating ability alone will appear in the tree. The features which dominate usually represent the condition of wind turbine blade.

From figure $6, \mathrm{X} 33$ is the most dominating feature followed by X34, X35, X36 and X32.

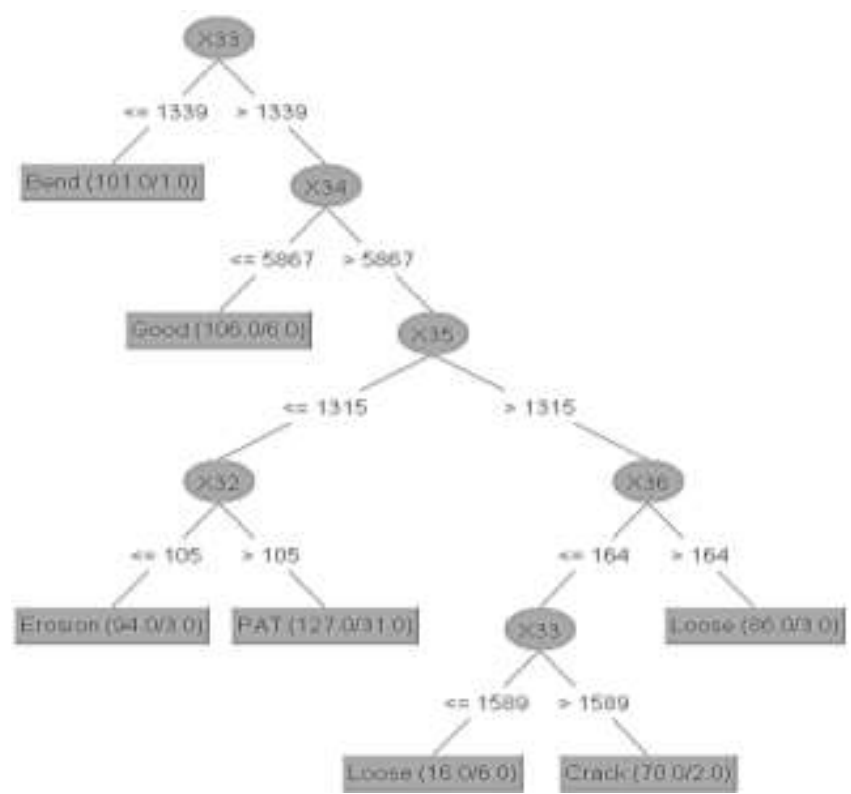

\section{Figure 6: J48 Tree Classification for Feature Selection}

V. FEATURE CLASSIFICATION

Next step after feature selection is feature classification. The chosen elements are to be classified utilizing the Nested Dichotomy classifier.

\section{Nested Dichotomy (ND)}

A basic approach to enhance the classification accuracy for problems with a little number of classes is to store twoclass models and re-use them in distinctive individuals of an ensemble of nested dichotomies. "An arrangement of nested dichotomies is a statistical model that is utilized to break down a multi-class issue into numerous two-class issues. The breakdown can be characterized by as a binary tree. Every hub of the tree stores an arrangement of class names, the equivalent preparing training data and a binary classifier. At the absolute starting point, the root hub contains the entire collections of the original class labels relating to the multi-class characterization issue. This set is then divided into two subsets. These two subsets of class names are treated as two 'meta' classes and a binary classifier is learned for predicting them. The training dataset is divided into two subsets relating to the two metaclasses and one subset of training data is viewed as the positive illustrations while the other subset of testing data is viewed as the negative cases. The two successor hubs of the root acquire the two subsets of the first class names with their corresponding training datasets and a tree is built by applying this process recursively. The procedure, at last, achieves a leaf hub if the hub contains one and only class name. It is clear that for any given c-class issue, the tree contains c leaf hubs (one for every class) and (c-1) interior hubs. Each interior hub contains a binary classifier. A good feature of utilizing a system of nested dichotomies for multiclass issues is that it yields class probability estimates in a direct manner. Frank and Kramer [17] inspected arbitrary from the space of every single conceivable tree by considering every tree with equivalent probability. 


\section{FAULT DIAGNOSIS OF WIND TURBINE BLADES USING HISTOGRAM FEATURES THROUGH NESTED DICHOTOMY CLASSIFIERS}

The determination of the tree structure will impact the characterization results. It signifies well to utilize all conceivable nested dichotomies for a given issue and mean their probability assessments to yield exact forecasts. For a c-class issue, the quantity of conceivable frameworks of nested dichotomies is $(2 c-3)$ !!. Subsequently, utilizing all conceivable nested dichotomies is not possible. Without former learning, any sampling plan that does not give special treatment to a specific class can be considered as a suitable competitor. The issue with arbitrary sampling based on uniform appropriation over trees is that the tree depth is just constrained by the number of classes, and deep trees can take quite a while to build.

\section{Class-Balanced Nested Dichotomies (CBND)}

The class-balanced nested dichotomy (CBND) technique depends on adjusting the number of classes at every hub. "Rather than testing from the space of every single conceivable tree (as in nested dichotomy), it is sampled from the space of every balanced tree. The benefit of this strategy is that the depth of the tree is ensured to be logarithmic in the number of classes.

The number of conceivable class- balanced nested dichotomies is clearly smaller than the aggregate number of nested dichotomies [18]. The relation characterizes the number of conceivable class-balanced trees.

$$
T(c)=\left\{\begin{array}{l}
1 / 2\left(\frac{c}{c / 2}\right) T\left(\frac{c}{2}\right) T\left(\frac{c}{2}\right), \text { If } c \text { is even } \\
\left(\begin{array}{c}
c \\
\frac{c+1}{2}
\end{array}\right) T\left(\frac{c+1}{c}\right) T\left(\frac{c-1}{c}\right), \text { If } c \text { is odd }
\end{array}\right.
$$

At every hub, the arrangement of classes is divided into equivalent size subsets (obviously, if the number of classes is odd, the size won't be precisely equivalent), and the base learning algorithm is used to the data comparing to these two subsets. The algorithm then recuses until one and only class is left out.

It is applied over and over with distinctive random number seeds to produce a committee of trees. It demonstrates that a non-paltry number of class-balanced nested dichotomy can be produced for classification issues with five or more classes. There is a further chance to enhance the training time for gatherings of nested dichotomies.

This is an added advantage of the class based nested dichotomy. There is a disadvantage with the class balanced methodology. Some multi-class issues are extremely unequal and a few classes are considerably more crowded than others.

In that case, a class-balanced tree does not imply that it is also data balanced. This can contrarily influence runtime if the base learning algorithm has time unpredictability more regrettable than linear in the number of instances.

\section{Data near Balanced Nested Dichotomy (DNBND)}

An easy algorithm called data near balanced nested dichotomy (DNBND) can be utilized as a substitute for the class balanced nested dichotomy. "Since this system violets the condition that the sampling plan should not be one-sided towards a specific class.

It arbitrary appoints classes to two subsets until the size of the training data in one of the subsets surpasses a large portion of the aggregate sum of training data at the hub. It is exceptionally fundamental to keep up a level of arbitrariness in the task of classes to subsets with a specific end goal to protect differing qualities in the committee of randomly generated systems of nested dichotomies [19].

On account of a skewed class appropriation, the base nested dichotomy algorithms' runtime is more poor than linear.

In that case, the number of instances is separated as uniformly as could be expected under the circumstances at every hub, in order to diminish the greatest measure of data considered at a hub as fast as would be prudent."

\section{RESULTS AND DISCUSSION}

The vibration signals were noted for good condition and faulty blade conditions using DAQ. "Totally 600 samples were collected; out of which 100 samples were from good condition blade. For different faults such as like blade bend, erosion, blade crack, hub-blade loose connection, pitch angle twist, 100 samples from every condition were noted.

J48 decision tree algorithm was used to select the best contributing histogram features from bin size 77 . From Figure 5 and 6 , the selected features are given as the input to the classifier to determine the classification accuracy with respect to faults created on the wind turbine blade.

From Figure 7, the class-balanced nested dichotomy (CBND) classifier gives the maximum classification accuracy of $93.17 \%$ when compared to other classifiers. In Nested Dichotomy, the default classifier is fixed to be rotation forest algorithm.

The confusion matrix of class-balanced nested dichotomy (CBND) is shown in Table 2. In confusion matrix, the diagonal element represents the correctly classified instance and the others are misclassified. From class-balanced nested dichotomy (CBND) classifier, the kappa statistics were found to be 0.918 . It is used to measure the arrangement of likelihood with the true class.

The mean absolute error was found to be 0.0438 . It is a measure used to measure how close forecasts or prediction are with the ultimate result. The root mean square error was found to be 0.1321 . It is a quadratic scoring rule which processes the average size of the error. The detailed class wise accuracy is shown in Table 3.

Out of 600 samples, 559 samples are correctly classified $(93.17 \%)$ and remaining 41 are misclassified $(6.83 \%)$. The time taken to build the model is about 0.29 seconds; hence, this can use in real time for the fault detection on the wind turbine blade.

Table 2: Confusion Matrix for Class-Balanced Nested Dichotomy (CBND)

\begin{tabular}{|l|l|l|l|l|l|l|}
\hline $\begin{array}{l}\text { Blade } \\
\text { condition } \\
\text { s }\end{array}$ & $\begin{array}{l}\text { Goo } \\
\mathrm{d}\end{array}$ & $\begin{array}{l}\text { Ben } \\
\mathrm{d}\end{array}$ & $\begin{array}{l}\text { Crac } \\
\mathrm{k}\end{array}$ & $\begin{array}{l}\text { Erosio } \\
\mathrm{n}\end{array}$ & $\begin{array}{l}\text { Loos } \\
\mathrm{e}\end{array}$ & $\begin{array}{l}\mathrm{PA} \\
\mathrm{T}\end{array}$ \\
\hline Good & $\mathbf{9 6}$ & 0 & 1 & 0 & 3 & 0 \\
\hline Bend & 0 & $\mathbf{1 0 0}$ & 0 & 0 & 0 & 0 \\
\hline Crack & 0 & 0 & $\mathbf{8 6}$ & 3 & 5 & 6 \\
\hline Erosion & 0 & 1 & 3 & $\mathbf{9 5}$ & 0 & 1 \\
\hline Loose & 5 & 1 & 3 & 0 & $\mathbf{9 1}$ & 0 \\
\hline PAT & 0 & 0 & 7 & 2 & 0 & $\mathbf{9 1}$ \\
\hline
\end{tabular}


Table 3: Classwise Accuracy of Class-Balanced Nested Dichotomy (CBND)

\begin{tabular}{|l|l|l|l|l|l|l|l|l|}
\hline $\begin{array}{l}\text { Clas } \\
\mathrm{s}\end{array}$ & $\begin{array}{l}\mathrm{TP} \\
\text { Ra } \\
\text { te }\end{array}$ & $\begin{array}{l}\text { FP } \\
\text { Rat } \\
\mathrm{e}\end{array}$ & $\begin{array}{l}\text { Precis } \\
\text { ion }\end{array}$ & $\begin{array}{l}\text { Rec } \\
\text { all }\end{array}$ & $\begin{array}{l}\text { F- } \\
\text { Meas } \\
\text { ure }\end{array}$ & $\begin{array}{l}\text { M } \\
\text { CC }\end{array}$ & $\begin{array}{l}\text { RO } \\
\text { Ar } \\
\text { ea }\end{array}$ & $\begin{array}{l}\text { PR } \\
\text { Ar } \\
\text { ea }\end{array}$ \\
\hline $\begin{array}{l}\text { Goo } \\
\text { d }\end{array}$ & $\begin{array}{l}0 . \\
96\end{array}$ & $\begin{array}{l}0.0 \\
14\end{array}$ & 0.931 & $\begin{array}{l}0.9 \\
6\end{array}$ & 0.941 & $\begin{array}{l}0.9 \\
29\end{array}$ & $\begin{array}{l}0.9 \\
99\end{array}$ & $\begin{array}{l}0.9 \\
94\end{array}$ \\
\hline $\begin{array}{l}\text { Ben } \\
\text { d }\end{array}$ & 1 & 0 & 1 & 1 & 0.990 & $\begin{array}{l}0.9 \\
88\end{array}$ & 1 & 1 \\
\hline $\begin{array}{l}\text { Crac } \\
\text { k }\end{array}$ & $\begin{array}{l}0 . \\
86\end{array}$ & $\begin{array}{l}0.0 \\
30\end{array}$ & 0.851 & $\begin{array}{l}0.8 \\
6\end{array}$ & 0.856 & $\begin{array}{l}0.8 \\
27\end{array}$ & $\begin{array}{l}0.9 \\
86\end{array}$ & $\begin{array}{l}0.9 \\
31\end{array}$ \\
\hline $\begin{array}{l}\text { Eros } \\
\text { ion }\end{array}$ & $\begin{array}{l}0 . \\
95\end{array}$ & $\begin{array}{l}0.0 \\
10\end{array}$ & 0.950 & $\begin{array}{l}0.9 \\
50\end{array}$ & 0.950 & $\begin{array}{l}0.9 \\
40\end{array}$ & $\begin{array}{l}0.9 \\
96\end{array}$ & $\begin{array}{l}0.9 \\
85\end{array}$ \\
\hline $\begin{array}{l}\text { Loos } \\
\text { e }\end{array}$ & $\begin{array}{l}0 . \\
91\end{array}$ & $\begin{array}{l}0.0 \\
18\end{array}$ & 0.909 & $\begin{array}{l}0.9 \\
1\end{array}$ & 0.905 & $\begin{array}{l}0.8 \\
86\end{array}$ & $\begin{array}{l}0.9 \\
95\end{array}$ & $\begin{array}{l}0.9 \\
77\end{array}$ \\
\hline PAT & $\begin{array}{l}0 . \\
91\end{array}$ & $\begin{array}{l}0.0 \\
12\end{array}$ & 0.938 & $\begin{array}{l}0.9 \\
1\end{array}$ & 0.918 & $\begin{array}{l}0.9 \\
03\end{array}$ & $\begin{array}{l}0.9 \\
91\end{array}$ & $\begin{array}{l}0.9 \\
72\end{array}$ \\
\hline
\end{tabular}

From class wise accuracy of class-balanced nested dichotomy (CBND) (Table 3), the properties like true positive rate (TP), false positive rate (FP), precision, recall, F-Measure, Matthews correlation coefficient (MCC), precision-recall curve (PRC) area, receiver operating characteristics (ROC) area are determined. TP is also called as sensitivity which used to predict the ratio of positives which are correctly classified as faults. FP is commonly described as false alarm in which the result that shows a given fault condition has been achieved, when it really has not been achieved [20-23]. The true positive (TP) rate should be close to 1 and the false positive (FP) rate should be close to 0 to propose the classifier is a better classifier for the problem classification [24-27]. In the class-balanced nested dichotomy (CBND), it shows that the TP near to 1 and FP close to 0 , then one can predict that the classifier we build for the particular problem is very much effective for the fault diagnosis problem."

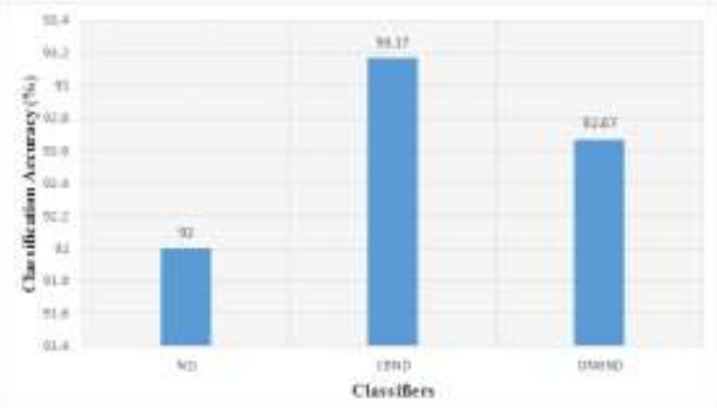

Figure 7: Overall Classification Accuracy of the Classifiers

Precision is the ratio of correctly classified instances for those instances that have been classified as positive. "Recall is merely equal to sensitivity in which the information retrieval is the fraction of the faults that are relevant to the query that are successfully retrieved. F-measure is defined as the equivalent resistance formed by sensitivity and precision positioned in parallel phase [28-32]. Matthews's correlation coefficient (MCC) is used to measure of the class of binary classifications. The MCC is in essence a correlation coefficient between the observed and predicted binary classifications; it returns a value between -1 and +1 [33-37]. Precision-recall curve (PRC) area is used to predict the classification properties using precision and recall values
[38-44]. It is more or less similar to receiver operating characteristics (ROC). ROC is a graphical representation that demonstrates the performance of a classifier as its discrimination threshold is varied."

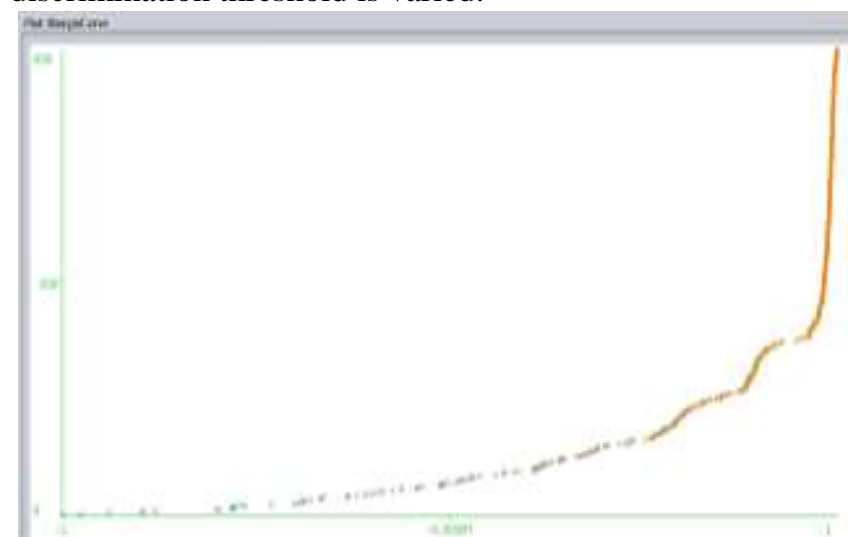

Figure 8: Margin Curve for Class-Balanced Nested Dichotomy (CBND)

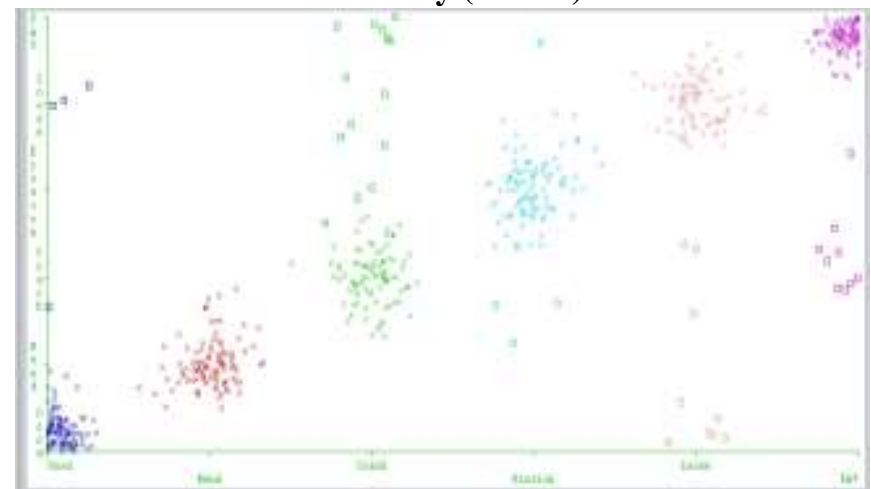

Figure 9: Classifier Errors (Classification vs. Misclassification)

VII. CONCLUSION

The wind turbine is very important in the creation of wind energy in everyday life. This paper displayed an algorithmic based clarification of vibration signals for the evaluation of the wind turbine blade conditions. From the acquired vibration data, three model was developed using data modelling technique. These models were tested in 10-fold cross validation. All the classifiers were compared with respect to their types and maximum correctly classified instances were found to be $93.17 \%$ for class-balanced nested dichotomy (CBND). The error rate is relatively less and may be considered for the blade fault diagnosis. Hence, the class balanced nested dichotomy (CBND) algorithm can be practically used for the condition monitoring of wind turbine blade to reduce the downtime and to provide more wind energy. The methodology and algorithm suggested in this paper can be potentially used for any kind of wind turbine blade to diagnose the blade fault with minimal modification.

\section{REFERENCES}

1. Herbert GJ, Iniyan S, Sreevalsan E, Rajapandian S. A review of wind energy technologies. Renewable and sustainable energy Reviews. 2007 Aug 31; 11(6):111745.

2. Ciang CC, Lee JR, Bang HJ. Structural health monitoring for a wind turbine system: a review of 

DICHOTOMY CLASSIFIERS

damage detection methods. Measurement Science and Technology. 2008 Oct 13; 19(12):122001.

3. Muralidharan V, Sugumaran V. A comparative study of Naïve Bayes classifier and Bayes net classifier for fault diagnosis of monoblock centrifugal pump using wavelet analysis. Applied Soft Computing. 2012 Aug 31; 12(8):2023-9.

4. Kamp LM, Smits RE, Andriesse CD. Notions on Netherlands and Denmark. Energy policy. 2004 Sep 30; 32(14):1625-37.

5. Kusiak A, Verma A. A data-driven approach for monitoring blade pitch faults in wind turbines. Sustainable Energy, IEEE Transactions on. 2011 Jan; 2(1):87-96.

6. Abouhnik A, Albarbar A. Wind turbine blades condition assessment based on vibration measurements and the level of an empirically decomposed feature. Energy Conversion and Management. 2012 Dec 31; 64:606-13.

7. Godwin JL, Matthews P. Classification and detection of wind turbine pitch faults through SCADA data analysis. IJPHM Special Issue on Wind Turbine PHM (Color). 2013:90.

8. Frost SA, Goebel K, Obrecht L. Integrating Structural Health Management with Contingency Control for Wind Turbines. IJPHM Special Issue on Wind Turbine PHM (Color). 2013:11.

9. Chen B, Matthews PC, Tavner PJ. Wind turbine pitch faults prognosis using a-priori knowledge-based ANFIS. Expert Systems with Applications. 2013 Dec 1; 40(17):6863-76.

10. Bouzid OM, Tian GY, Cumanan K, Moore D, Structural Health Monitoring of Wind Turbine Blades: Acoustic Source Localization Using Wireless Sensor Networks, Journal of Sensors, 2014 Dec 29.

11. Lee JK, Park JY, Oh KY, Ju SH, Lee JS. Transformation algorithm of wind turbine blade moment signals for blade condition monitoring. Renewable Energy. $2015 \mathrm{Jul}$ 31; 79:209-18.

12. Herath MT, Lee AK, Prusty BG. Design of shapeadaptive wind turbine blades using Differential Stiffness Bend-Twist coupling. Ocean Engineering. 2015 Feb 1; 95:157-65.

13. Liu C, Jiang D, Chu F. Influence of alternating loads on nonlinear vibration characteristics of cracked blade in rotor system. Journal of Sound and Vibration. 2015 Sep 29; 353:205-19.

14. Joshuva A, Sugumaran V. Fault Diagnostic Methods for Wind Turbine: A Review. ARPN Journal of Engineering and Applied Sciences. 2016 Apr: 11(7):4654-68.

15. Joshuva A, Sugumaran V. A data driven approach for condition monitoring of wind turbine blade using vibration signals through best-first tree algorithm and functional trees algorithm: A comparative study. ISA transactions. 2017 Mar 1; 67:160-172.

16. Joshuva A, Sugumaran V, Amarnath M. Selecting kernel function of Support Vector Machine for fault diagnosis of roller bearings using sound signals through histogram features. International Journal of Applied Engineering Research. 2015; 10(68):482-487.

17. Frank, E. \& Kramer, S., Ensembles of nested dichotomies for multi-class problems. In: Proc. Int. Conf. on Machine Learning. ACM, 2004, pp.305 - 312.

18. Dong L. L, Frank E \& Kramer S. (2005) Ensembles of Balanced Nested Dichotomies for Multi-Class Problems, Knowledge Discovery in Databases: PKDD 2005, 2005, pp. 84-95.

19. Joshuva A, Sugumaran V. Wind turbine blade fault diagnosis using vibration signals through decision tree algorithm. Indian Journal of Science and Technology. 2016 Dec 29; 9(48):1-7. learning applied to wind turbine development in the

20. Burke DS, Brundage JF, Redfield RR, Damato JJ, Schable CA, Putman P, Visintine R, Kim HI. Measurement of the false positive rate in a screening program for human immunodeficiency virus infections. New England Journal of Medicine. 1988 Oct 13; 319(15):961-4.

21. Peck R, Devore JL. Statistics: The exploration \& analysis of data. Cengage Learning; 2011.

22. Joshuva A, Sugumaran V. A comparative study of Bayes classifiers for blade fault diagnosis in wind turbines through vibration signals. Struct Durab Health Monit (SDHM). 2017; 12(1):69-90.

23. Matthews BW. Comparison of the predicted and observed secondary structure of T4 phage lysozyme. Biochimica et Biophysica Acta (BBA)-Protein Structure. 1975 Oct 20; 405(2):442-51.

24. Joshuva A, Sugumaran V. A Comparative Study for Condition Monitoring on Wind Turbine Blade using Vibration Signals through Statistical Features: A Lazy Learning Approach. International Journal of Engineering and Technology. 2018; 7(4.10):190-196.

25. Joshuva A, Sugumaran V. A Machine Learning Approach For Condition Monitoring Of Wind Turbine Blade Using Autoregressive Moving Average (ARMA) Features Through Vibration Signals: A Comparative Study. Progress in Industrial Ecology-An International Journal. 2018; 12(1/2):14-34.

26. Hall M, Frank E, Holmes G, Pfahringer B, Reutemann P, Witten IH. The WEKA data mining software: an update. ACM SIGKDD explorations newsletter. 2009 Nov 16; 11(1):10-8.

27. Joshuva A, Sugumaran V. Classification of various wind turbine blade faults through vibration signals using hyperpipes and voting feature intervals algorithm. International Journal of Performability Engineering. 2017; 13(3):247-258.

28. Joshuva A, Sugumaran V. Failure Analysis on Wind Blade Using Vibration Signals and Classifying the Failures Using Logit Boost Algorithm. International Journal of Control Theory and Applications. 2016; 9(52):225-234.

29. More Vasudha, Panditrao Harshal, Joshuva A, Sugumaran V. Effect of Sampling Frequency and Sample Length on Fault Diagnosis of Wind Turbine Blade. Pakistan Journal of Biotechnology. 2018; 15(Special Issue ICRAME 17):14-17.

30. Joshuva A, Sugumaran V. Fault Diagnosis of Wind Turbine Blade Using Vibration Signals through J48 Decision Tree Algorithm and Random Tree Classifier. International Journal of Control Theory and Applications. 2016; 9(52):249-258.

31. Joshuva A, Sugumaran V. Multiclass Classifier Approach for Fault Diagnosis of Wind Turbine Blade Using Vibration Signals through Statistical Analysis. International Journal of Control Theory and Applications. 2016; 9(52):235-247.

32. Davis J, Goadrich M. The relationship between Precision-Recall and ROC curves. In Proceedings of the 23rd international conference on Machine learning 2006 Jun 25 (pp. 233-240). ACM.

33. Joshuva A, Sugumaran V, Amarnath M, Lee SK Remaining Life-Time Assessment of Gear Box Using Regression Model. Indian Journal of Science and Technology. 2016 Dec 28; 9(47):1-8.

34. Joshuva A, Sugumaran V. A Study of Various Blade Fault Conditions on a Wind Turbine Using Vibration Signals through Histogram Features. Journal of 
Engineering Science and Technology. 2018 Jan; 13(1):102-121.

35. Manju BR, Joshuva A, Sugumaran V. A Data Mining Study for Condition Monitoring on Wind Turbine Blades Using Hoeffding Tree Algorithm Through Statistical and Histogram Features. International Journal of Mechanical Engineering and Technology. 2018; 9(1):1061-1079.

36. Joshuva A, Sugumaran V. Wind Turbine Blade Fault Diagnosis Using Vibration Signals and Statistical Features through Nested Dichotomy Classifiers. International Journal of Pharmacy and Technology. 2017 Apr;9(1): 29147-29164.

37. Joshuva A, Sugumaran V. Fault Diagnosis for Wind Turbine Blade through Vibration Signals Using Statistical Features and Random Forest Algorithm. International Journal of Pharmacy and Technology. 2017 Apr; 9(1): 28684-28696.

38. Deenadayalan, Sivakumar, S., Vishnuvardhan R., Sathish Kumar R. Fabrication and Characterisation of B-H-G Fiber with Teak Wood Particles Reinforced Hybrid Composite. International Journal of Engineering and Technology. 2018; 7(2.31): 208-211.

39. Sivakumar S, Dhanalakshmi V, Vishuvardhan R Extraction of Subtractive Features of Prismatic Parts from STEP File for CAD/CAM Integration. Journal of Science and Technology, Malaysia. 2019 Jan; 27 (1): 343-356.

40. Vishnuvardhan R, Pooranam N, Sivakumar S, Vignesh T. Producing Electrical Energy from Light Intensity, Design Traking System by Dual Axis Method. International Journal of Mechanical and Production Engineering Research and Development (IJMPERD). 2019; Jan; 9: 503-509.

41. Joshuva A, Sugumaran V. Improvement in wind energy production through condition monitoring of wind turbine blades using vibration signatures and ARMA features: a data-driven approach. Progress in Industrial Ecology, an International Journal. 2019 Jun 21; 13(3):207-31.

42. Joshuva A, Sugumaran V. Selection of a meta classifierdata model for classifying wind turbine blade fault conditions using histogram features and vibration signals: a data-mining study. Progress in Industrial Ecology, an International Journal. 2019 Jun 21; 13(3):232-51.

43. Joshuva A, Sugumaran V. Crack Detection and Localization on Wind Turbine Blade Using Machine Learning Algorithms: A Data Mining Approach. Struct Durab Health Monit (SDHM). 2019; 13(2):181-203.

44. Joshuva A, Sugumaran V. Fault Diagnosis and Localization of Wind Turbine Blade [dissertation], Vellore Institute of Technology, Chennai Campus. 2018. 